\title{
Exploiting Overlapping Channels for Minimum Power Configuration in Real-Time Sensor Networks
}

\author{
Xiaodong Wang ${ }^{1}$, Xiaorui Wang ${ }^{1}$, Guoliang Xing ${ }^{2}$, and Yanjun Yao ${ }^{1}$ \\ ${ }^{1}$ Department of EECS, University of Tennessee, Knoxville, TN 37996 \\ ${ }^{2}$ Department of CSE, Michigan State University, MI 48824 \\ \{xwang33,xwang,yyao9\}@utk.edu,glxing@cse.msu.edu
}

\begin{abstract}
Multi-channel communications can effectively reduce channel competition and interferences in a wireless sensor network, and thus achieve increased throughput and improved end-to-end delay guarantees with reduced power consumption. However, existing work relies only on a small number of orthogonal channels, resulting in degraded performance when a large number of data flows need to be transmitted on different channels. In this paper, we conduct empirical studies to investigate the interferences among overlapping channels. Our results show that overlapping channels can also be utilized for improved real-time performance if the node transmission power is carefully configured. In order to minimize the overall power consumption of a network with multiple data flows under end-to-end delay constraints, we formulate a constrained optimization problem to configure the transmission power level for every node and assign overlapping channels to different data flows. Since the optimization problem has an exponential computational complexity, we then present a heuristic algorithm designed based on Simulated Annealing to find a suboptimal solution. Our empirical results on a 25-mote testbed demonstrate that our algorithm achieves better real-time performance and less power consumption than two baselines including a scheme using only orthogonal channels.
\end{abstract}

\section{Introduction}

Many wireless sensor network (WSN) applications must address multiple stringent design constraints such as energy consumption and end-to-end communication delay. Energy has long been treated as the primary optimization goal for battery-powered wireless sensor nodes. With lower energy consumption, a network can achieve a longer lifetime. In addition to periodic sleeping, one of the effective ways to reduce node energy consumption is to lower its radio transmission power. This can be supported by the existing sensor mote hardware. For example, the CC2420 radio chip [1] used in many mote hardware platforms has 31 different transmission power levels. However, reducing transmission power may lead to unreliable wireless links and cause increased number of retransmissions. As a result, it may lead to poor guarantees of other important design 
constraints such as end-to-end delay, as many WSN applications require information to be transmitted from sources to sinks within an application-specified deadline. Therefore, transmission power must be carefully configured in order to meet the desired constraints of a WSN. High transmission power may improve the quality of a single wireless link but may lead to increased power consumption, stronger interferences to other links, and reduced network capacity [2].

The emergence of multi-channel mote hardware has made it possible to achieve improved throughput and delay guarantees with reduced transmission power, by using different channels on different nodes, leading to less channel competition and interference in the network. For example,the CC2420 radio chip provides 16 wireless channels with radio frequency from 2,400 to $2,483 \mathrm{MHz}$. As a result, multi-channel communication protocols have been proposed for WSNs to improve the performance of traditional single-channel protocols commonly used in WSNs. Based on the channel allocation scheme, existing multi-channel protocols can be categorized to two classes: node-based and flow-based. In node-based protocols, channels are assigned to different nodes locally to minimize interferences. For example, several node-based multi-channel MAC protocols [3][4] have been proposed to improve network throughput for WSNs. However, a major problem for node-based assignment is that nodes usually need to switch channels in order to receive data from and transmit to different neighbors, which may result in a high overhead, in terms of latency and power consumption. In flowbased protocols, the nodes in the same data flow are assigned the same channel so that frequent channel switching is avoided. For example, a flow-based multichannel real-time communication protocol, known as MCRT [5], has recently been presented to allow different data flows to transmit on different channels for improved end-to-end real-time guarantees with reduced power consumption. MCRT has been demonstrated to outperform node-based schemes by having a smaller deadline miss ratio and lower power consumption.

While multi-channel communications have shown great promise, recent studies (e.g., [6]) conducted experiments on Micaz hardware to investigate multichannel realities in wireless sensor networks. An important reality reported is that the number of orthogonal channels is actually small for the existing mote hardware. Accordingly, it has been suggested that a practical multi-channel communication protocol should only rely on a small number of non-adjacent orthogonal channels, because adjacent overlapping channels may have undesired inter-channel interferences. For example, at most, only 8 channels out of the 16 channels provided by the CC2420 radio chip can be used as orthogonal channels [6], resulting in the waste of half of the available wireless channel resources. While 8 channels may be enough for some WSN applications, using only orthogonal channels has limited the further improvement of network throughput, real-time performance, and power optimization in the commonly used many-to-one traffic pattern, where the number of data flows can be large in a network.

In this paper, we propose to utilize adjacent overlapping channels to configure power and channels for a WSN to achieve improved real-time performance and reduced power consumption. The power and channel configuration problem is de- 
fined as follows: Given a WSN with multiple data flows from different sources to the base station, our goal is to assign channels (including overlapping channels) to the data flows and determine a transmission power level for every node in the network, such that the overall (transmission) power consumption of the network can be minimized while the average end-to-end delay of each data flow can be guaranteed to stay within a deadline. In order to motivate our work, we first conduct hardware experiments to investigate the interferences among overlapping channels. We then use empirical studies for overlapping channel modeling. Based on our models, we formulate the power and channel configuration problem as a constrained optimization problem, with power minimization as the objective and the end-to-end delay as the constraints. Since it is cost-prohibitive to find the optimal solution, we propose a heuristic algorithm based on Simulated Annealing to find a suboptimal solution. Finally, we conduct experiments on a 25-mote testbed to show that our configuration outperforms two baseline solutions.

To our best knowledge, this paper presents the first study of utilizing overlapping channels to achieve minimum transmission power configuration and guaranteed real-time performance in wireless sensor networks. Specifically, the contributions of this paper are four-fold.

- We conduct empirical studies to investigate the interferences among overlapping channels. Our results show that overlapping channels can also be utilized for improved real-time performance if the node transmission power is carefully configured.

- We establish an empirical model between received signal strength (RSS) and transmission power level for overlapping channels. Based on the RSS model, we model the relationship between packet reception ratio (PRR) and RSS to account for the interferences from overlapping channels.

- We formulate the power and channel configuration problem as a constrained optimization problem. Since the problem has an exponential computational complexity, we then present a heuristic algorithm designed based on Simulated Annealing (SA) to find a suboptimal solution, which can be executed periodically or in an on-demand fashion.

- We implement our algorithm on the Tmote hardware and conduct experiments on a 25-mote testbed. Our results demonstrate that our algorithm can reduce both end-to-end communication delay and overall transmission power consumption, compared with two baselines. The first baseline conducts the same optimization using only orthogonal channels. The second baseline uses SA to find the desired power level but randomly assigns overlapping channels.

The rest of paper is organized as follows. Section 2 highlights the distinction of our work by discussing the related work. Section 3 presents empirical studies to motivate this work and build models for overlapping channels. Section 4 introduces the formulation of our optimization problem with real-time performance analysis. Section 5 presents the algorithm we used to solve the optimization problem. In Section 6, we present the empirical results of our algorithm. Section 7 concludes the paper. 


\section{Related Work}

Recent studies have proposed to use partially overlapping channels (POC) in wireless mesh networks. Liu et al. [7] propose a channel allocation scheme for link scheduling, which takes advantages of POC to obtain better throughput for mesh networks. Feng et al. [8] establish an interference model for POC-based wireless networks, and use numeric methods to improve overall network capacity. A linear model for channel assignment, which uses a channel overlapping matrix and mutual interference matrices to model POC channels, has been proposed in [9]. However, no detailed study has been performed to utilize overlapping channels in wireless sensor networks for improved real-time performance. A coarse-grained channel assignment policy for WSNs is proposed in [6], which allocates nonoverlapping channels to disjoint trees and exploits parallel transmissions among trees. In this paper, we propose to utilize overlapping channels to configure power and channels for a WSN to achieve better real-time performance and energy efficiency.

Several projects have studied received signal strength (RSS) and its utilization in WSNs. Sha et al. [10] establish a model between the RSS and transmission power in a single channel. Demirbas et al. [11] present a robust and lightweight solution for sybil attack problem based on the received signal strength indicator (RSSI) readings of messages in WSNs. However, none of these projects study the relationship between RSS and transmission power in multi-channel WSNs. In this paper, we establish the RSS model for overlapping channels and formulate a transmission power minimization problem based on the models.

Many real-time communication protocols have been proposed for wireless sensor and ad hoc networks. A comprehensive review of real-time communication in WSNs is presented in [12]. At the MAC layer, Implicit EDF [13] is a collisionfree real-time scheduling scheme by exploiting the periodicity of WSN traffic. At higher layers, SPEED [14] achieves desired end-to-end communication delays by enforcing a uniform communication speed throughout the network. However, most of the existing real-time protocols do not take advantage of the capability of multi-channel communications available in today's mote hardware. In our work, we address the problem of utilizing overlapping channels for improved real-time performance.

Different from all the aforementioned work that handles real-time guarantees, partially overlapping channels, and energy efficiency in isolation, our design utilizes overlapping channels available on existing sensor mote hardware to achieve more energy-efficient transmission for multi-channel WSNs under real-time constraints.

\section{Empirical Modeling of Overlapping Channels}

Previous work [6] has reported that adjacent overlapping channels have undesired inter-channel interferences. In this section, we first investigate the impacts of overlapping channels on packet reception ratio (PRR) of link to motivate our work. We then extend existing work to establish an empirical model between 
RSS and transmission power level for overlapping channels. Based on the RSS model, we derive a PRR model to account for the interferences from overlapping channels.

\subsection{Case Study for Motivation}

In this section, a case study is performed with two pairs of nodes, which compose two one-hop communication links. In the experiment, one pair of motes performs as the transmission pair by using channel 16. The other pair of motes, acting as the jammer pair, uses an adjacent channel, 15, to communicate. The transmissions of these two pairs are synchronized. The transmission power of the transmission pair is fixed at power level 15, while the transmission power of the jammer pair increases one level at a time from level 3 to level 31. One hundred packets are transmitted on both pairs at each power level. We calculate the PRR of each pair in this experiment under different transmission power levels of the jammer pair. The results are shown in Figure 1. From the results we can see that both the two pairs can achieve a good PRR when the jammer pair is using power levels 16 to 18 for transmission. When the jammer is using a lower power level, its communication does not incur much interference to the transmission pair. The transmission pair can reach a high PRR. When the jammer pair is using a higher power level to transmit, it can improve the packet reception ratio of its own communication, but incurs too much interference to the transmission pair, and so hurts the communication quality of the transmission pair. This experiment shows that given two communication links working on overlapping channels, we can achieve good quality for both transmissions if we carefully choose the transmission power.

\subsection{Overlapping Channel RSS Model}

As discussed in subsection 3.1, with careful selection of transmission power, two links working on adjacent channels can both achieve a high PRR. An approximate linear correlation between RSS and transmission power over a singlechannel single-hop link is reported in [10]. In this subsection, we extend the method proposed in [15] to study the relationship between RSS and transmission power in the scenario where a sender and a receiver are working on adjacent
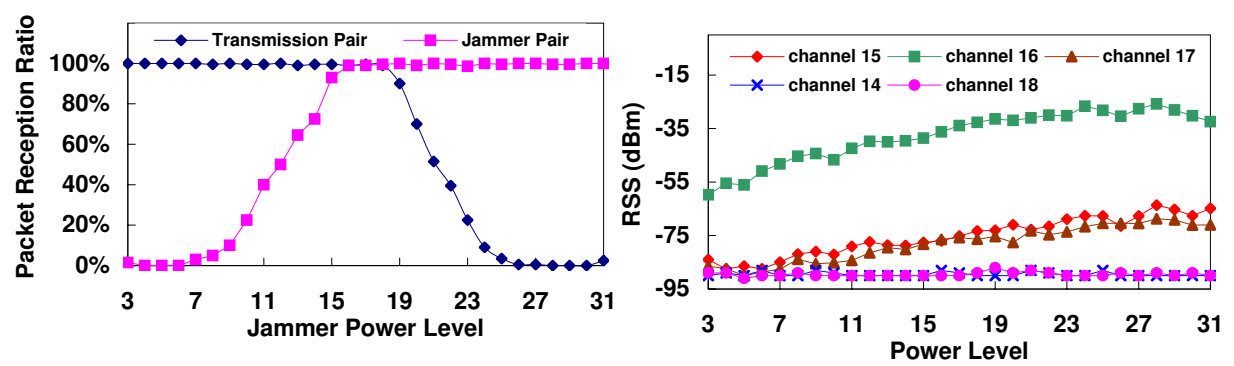

Fig. 1. Packet reception ratio vs. jammer power level. (Jammer uses channel 15 and sender uses channel 16.)

Fig. 2. RSS vs. power level on different channels. (Sender uses channel 16.) 
channels. We conduct the signal strength detection experiment on a single link to explore the overlapping channel property.

Our experiment uses two Tmote Invent motes. One mote acts as the sender and the other as the RSS sensor. In the experiment, the sender continuously broadcasts packets at a rate of 100 packets per second. The RSS sensor continuously collects the received signal strength by periodically reading the value of the Received Signal Strength Indicator (RSSI) on the mote at a rate of 100 times per second. After sending 100 packets at one power level, the sender lowers its transmission power by 1 level, starting from level 31 to level 3 . We first filter out the noise value by using the noise floor threshold we collected before the experiment and then calculate the average RSS value. We test various combinations of sending and receiving channels in this experiment.

Figure 2 shows the result when the sender is using channel 16. We can see that when the sender is using channel 16 for broadcasting, the RSS values sensed on the two adjacent channels, channel 15 and channel 17 show highly linear correlation with sender's transmission power. However, no clear RSS reading is sensed on channel 14 or channel 18. In addition, the results show an approximate linear increasing trend when the sender and the RSS sensor are using the same channel, channel 16. Previous work [10] presents the empirical single-channel RSS-Power model as:

$$
R S S\left(v, u, p_{u}\right)=A_{u, v} \times p_{u}+B_{u, v},
$$

where $v$ is the receiving node, $u$ is the sending node, and $p_{u}$ is the transmission power at $u$. $A$ and $B$ are two parameters of the model, which can be calculated by applying linear curve fitting to the sampled data. Note that distance is not considered in Equation 1 because the RSS value is dynamically measured between each given pair of sender and sensor.

Based on the observation of similar linear pattern when the sender and receiver are using adjacent channels, we re-establish the empirical RSS-Power model under multi-channel conditions as:

$$
R S S\left(v, u, p_{u}, c_{v}, c_{u}\right)=A_{u, v, c_{v}, c_{u}} \times p_{u}+B_{u, v, c_{v}, c_{u}},
$$

where $c_{u}$ is the transmitting channel for sender $u$ and $c_{v}$ is the listening channel for receiver $v$. $A$ and $B$ are the two model parameters, which are usually decided by the the application environment, such as network condition and communication distance. A similar model is reported in [15]. Our model uses a simplified threshold filter to filter out the noise for faster runtime processing, while a CPM noise filter is used in [15].

Using linear curve fitting to establish our model gives us a fast way to accomplish the model establishment, depending on the number of sampling points we need. One second is required for the signal strength readings for each power level in the model, as explained previously. If we use 5 power levels to build the model, the total time for the model establishment is only 5 seconds. Therefore, our model can be promptly rebuilt at runtime to adapt to environmental or temporal variations of network conditions. Also, the overlapping channel RSS model of every node in a less dense network (e.g.[16]) can be quickly established. 


\subsection{Packet Reception Ratio}

Packet reception ratio (PRR) is the probability that a packet can be received successfully. Higher transmission power can provide a higher Signal to Interference and Noise Ratio (SINR) over the link, which leads to a higher PRR. However, with higher transmission power, the communication at the current link could significantly interfere with another link's communication as shown in subsection 3.1. In this section, we conduct an experiment to study the relationship between PRR and SINR. With an understanding of this relationship, we can find the appropriate transmission power range to reach a required SINR value for a desired PRR value.

In the experiment, we use three Tmote Invent motes, one as the receiver $C$ and the other two as transmitting motes, $A$ and $B$. All of the three motes use the same channel. This experiment consists of three rounds. In the first round, we only turn on motes $A$ and $C$. We use $A$ to transmit multiple packets to receiver $C$ and calculate the average received signal strength of the packets, denoted as $R S S(A, C)$. In the second round, we turn $A$ off and use $B$ to transmit multiple packets to the receiver. We then calculate the average received signal strength of $B$, denoted as $R S S(B, C)$. In the third round, all the three motes are turned on. Both $A$ and $B$ transmit multiple packets to receiver $C$. The transmissions are synchronized. We calculate the PRR for A's transmission, denoted as $\operatorname{PRR}(A, C)$.

Considering B's transmission as the interference to A's transmission, we can calculate the SINR value for $A$ 's transmission as follows:

$$
\operatorname{SINR}(A, C)_{d B}=R S S(A, C)-10 \log _{10}\left(10^{\frac{R S S(B, C)}{10}}+10^{\frac{N}{10}}\right)
$$

where $N$ is the noise floor value, which is collected before the experiment. Equation 3 is derived from the SINR equation from [17]. By doing the above three steps and applying the equation, we get a PRR-SINR pair. We repeat the experiment with different distances from $B$ to receiver $C$ and different transmission power levels used by $A$ and $B$ to create different SINR values at the receiver. Figure 3 shows the PRR-SINR relationship in our experiment. When the SINR value is greater

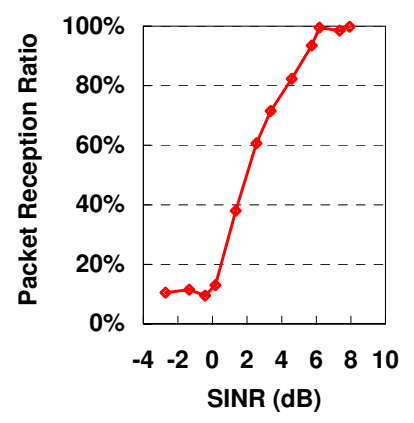

Fig. 3. PRR vs. SINR. than $6 \mathrm{~dB}$, the PRR is almost $100 \%$. Therefore, in order to achieve a good packet reception ratio in this sepcific experiment, e.g., 90\%, we need to choose a transmission power that can provide a strong enough received signal strength leading to an SINR value of more than $6 \mathrm{~dB}$.

In order to apply this experimental approach to multi-channel networks, we extend the PRR-SINR relationship by incorporating the channel information to it. We use $\left(S I N R_{v}, c_{v}, P R R_{v}\right)$, to denote the PRR-SINR-Channel relationship between node v's packet reception ratio and the corresponding SINR value in 
channel $c_{v}$. With this extension, we can obtain the required SINR value for a good PRR on a desired channel.

\section{Minimum Transmission Power Configuration}

In this section, we first formulate the power and channel configuration problem. We then analyze the node transmission delay in the network.

\subsection{Problem Formulation}

We assume the network has the common many-to-one traffic pattern [18][19], which is composed of multiple sources, some relay nodes and one base station. Each source generates a data flow to the base station. All the flows are assumed to be disjoint, since disjoint paths are widely used in multi-path routing to enhance the system's fault-tolerance [5][20]. The data generated at the source are assumed to follow a uniform random distribution [21]. We also assume that the base station is a super node with multiple radios such that it can work on several different frequencies at the same time. The channel allocation in our network is flow-based, which means all nodes in the same flow work on the same channel. Our goal is to minimize the total transmission power consumption under the constraint that the end-to-end delay of every flow in the given topology is constrained.

We first introduce the following notation:

- $G=(V, E)$, a directional graph denoting the network with $V$ nodes and $E$ edges (links).

$-f_{i}$, the data flow with the id number $i$.

- $D$, the delay constraint for each flow.

$-p_{u}$, transmitting power used by node $u$.

- $c_{u}$, the channel id used by node $u$, which is an integer number.

- $I(v)$, the interference node set of node $v$.

- $(u, v)$, a communication link in the graph, in which $u$ is the sending node and $v$ is the receiving node.

Given the notation above, we can formulate our minimization problem as:

$$
\min \sum_{v \in V:(u, v) \in G} p_{u} \times \frac{1}{P R R(u, v)}
$$

Subject to the constraints:

$$
\begin{gathered}
1 \leq c_{u} \leq n \quad \forall u \in G \\
c_{u}=c_{v} \quad \forall(u, v) \in G \\
\sum_{v \in f_{j}} \frac{1}{P R R(u, v)} \leq D \quad \forall j: 1 \leq j \leq m
\end{gathered}
$$


The inverse of $\operatorname{PRR}(u, v)$ in Equation 4 is the average transmission count required for a packet to be successfully received by node $v$ from node $u$. By multiplying $p_{u}$ and $\frac{1}{\operatorname{PRR}(u, v)}$, we obtain the transmission power consumption for one packet at node $u$. The objective of Equation 4 is to minimize the total transmission power consumption of all the nodes in the network. Equation 5 is the channel constraint, which confines that each node can only pick a channel from $n$ available channels. Equation 6 confines that all nodes in the same data flow must use the same channel. Equation 7 is the end-to-end delay constraint, which gives the limit of the end-to-end transmission count (including retransmissions at each node) for a packet in each flow. End-to-end transmission count is a commonly used metric to represent end-to-end delay as a higher transmission count leads to a longer end-to-end delay. Note that our minimization problem does not depend on the node duty cycle scheduling, so our work can be integrated with energy-efficient MAC protocols with periodic sleeping for further power savings at the cost of longer communication delays.

\subsection{Transmission Delay Analysis}

One way to analyze the node transmission delay in a WSN is to use the worst-case scenario, where we can assume that all the links in a neighborhood communicate at the same time, such that the most significant interference and delay are incurred. However, due to the lossy nature of wireless links, real-time communication protocols in WSNs are commonly designed to provide only soft probabilistic real-time guarantees [22][5]. In addition, the traffic patterns at different sources in many wireless sensor networks, such as surveillance applications [23], are usually independently random and unknown a priori. The chance for all the links in a neighborhood to transmit at exactly the same time is very small. Therefore, it is more meaningful to analyze the average case for WSNs. We modify our problem formulation as:

$$
\min \sum_{v \in V:(u, v) \in G} p_{u} \times \frac{1}{P R R_{a v g}(u, v)} .
$$

Correspondingly, the end-to-end delay constraint in Equation 7 is modified as:

$$
\sum_{v \in f_{j}} \frac{1}{P R R_{a v g}(u, v)} \leq D \quad \forall j: 1 \leq j \leq m
$$

where $P R R_{a v g}(u, v)$ is the average packet reception ratio at node $v$ when the generated traffic at the sender $u$ follows the random distribution.

Note that the probability for more than two nodes to transmit concurrently is small under the random traffic assumption. We assume that at most two nodes in the same interference range may transmit concurrently. We denote the probability that node $w$ 's transmission can interfere with node $u$ 's transmission as $P(u, w)$ and the packet reception ratio at the receiver $v$ from $u$ 's transmission under $w$ 's interference as $\operatorname{PRR}(u, v, w)$. We can use Equation 10 to estimate 


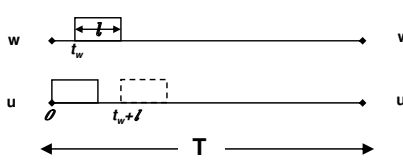

(a)

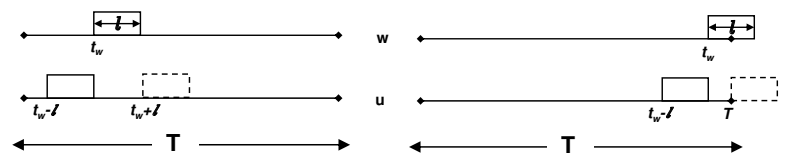

(b)

(c)

Fig. 4. Probability of packet collision when two nodes have independently random traffic.

the average transmission count for node $u$ to successfully transmit a packet to $v$ when $u$ and $w$ follow the independent random traffic pattern.

$\frac{1}{P R R_{a v g}(u, v)}=\left(1-\sum_{w \in I(u)} P(u, w)\right) \frac{1}{P R R(u, v, v)}+\sum_{w \in I(u)} P(u, w) \times \frac{1}{P R R(u, v, w)}$

In Equation 10, $\operatorname{PRR}(u, v, v)$ is the packet reception ratio at receiver $v$ when there is no interference to sender $u$ 's transmission.

Note that $P(u, w)$ in Equation 10 is the probability that node $u$ and node $w$ transmit packets concurrently. To derive $P(u, w)$, we assume that each source node has the same packet rate, 1 packet per $T$ seconds, with a packet length $l$. We denote the start time of the transmission at node $u$ and $w$ as $t_{u}$ and $t_{w}$, respectively. With the assumptions that the start time of every packet on the source node follows the uniform distribution and each intermediate node forwards packets immediately after receiving, we can calculate $P(u, w)$ as follows:

$$
P(u, w)= \begin{cases}\int_{0}^{l} \int_{0}^{t_{w}+l} \frac{1}{T^{2}} d t_{u} d t_{w} \quad \text { if } 0<t_{w} \leq l \\ \int_{l}^{T-l} \int_{t_{w}-l}^{t_{w}+l} \frac{1}{T^{2}} d t_{u} d t_{w} \text { if } l<t_{w} \leq T-l \\ \int_{T-l}^{T} \int_{t_{w}-l}^{T} \frac{1}{T^{2}} d t_{u} d t_{w} & \text { if } T-l<t_{w} \leq T\end{cases}
$$

Figure 4 illustrates the three cases in Equation 11. In the first case, when $t_{w} \leq l$, collision happens under the condition that $t_{u} \leq t_{w}+l$. In the second case, when $t_{w} \in(l, T-l]$, collision happens under the condition that $t_{u} \in\left(t_{w}-l, t_{w}+l\right)$. In the third case, when $t_{w} \in(T-l, T]$, collision happens only when $t_{u} \in\left(t_{w}-l, T\right]$. Note that the independently random traffic pattern assumption can be relaxed in the average PRR estimation. When the traffic pattern is not random, we can use empirical on-line testing to find the collision probability.

By integrating the three cases in Equation 11, we get the collision probability between two nodes in one period $T$ as:

$$
P(u, w)=\frac{l(2 T-l)}{T^{2}}
$$

Based on the models we established in Section 3, given a power level for each node in the network and a channel assignment to the data flows, we can compute the PRR for each receiving node under the interference from another 
node. By using Equation 10, we can derive the average transmission count for every node and further calculate the end-to-end delay of every flow, as well as the total system power consumption of the network for the given combination of power levels and channels. Our optimization objective is to find the combination with the least power consumption while the delay of every data flow is shorter than the given constraint.

\section{Algorithm Design}

The problem formulated in Section 4 is a complex combinatorial optimization problem with a huge search space. Suppose there are $j$ nodes forming $m$ flows in the network. The total available number of channels on the equipment is $n$. Each mote can use $k$ different power levels to transmit. The combinatorial search space has a size of $n^{m} \times k^{j}$. Therefore, we propose to use Simulated Annealing (SA) [24], a well-known meta-heuristic, to solve this problem. SA is commonly used to find suboptimal solutions when the search space is huge and discrete, which makes SA well suited for our problem because all possible configuration states are discrete, as the selection of channels and power levels are discrete numbers. Note that although the original SA algorithm is centralized, SA can be extended to run in a distributed way with slightly worse performance [25]. Therefore, our solution can also be extended to run on the sensor nodes in the network in a distributed way. The detailed extension is beyond the scope of this paper. In addition, please note that many real-world WSN applications adopt many-toone communication [26][27] for data collection, in which the sink is usually a sensor mote connected to the base station, such as a computer. The base station is commonly used to make centralized decisions for these applications.

Simulated Annealing is a probabilistic method for optimization problems. It transposes the process of the annealing of metal, in which the temperature of the metal is gradually decreased, to the solution search of the optimization problem. In each step, the algorithm considers some neighbor states of the current state, and chooses a valid neighbor state for the next state according to a probabilistic function established on the optimization goal. Two major parts of $S A$ are the neighbor state generation and the transition probability. The neighbor state generation scheme requires that every two adjacent states have a short distance. The transition probability is to decide whether the system should go to the next state, i.e., the neighbor state generated in the neighbor generation part.

The objective of our problem is to minimize the total transmission power consumption for the network under an end-to-end delay constraint. The configuration space consists of all the channel assignment and power configuration combinations. Based on a given channel and power configuration, the system proceeds to the next configuration by performing an elementary modification. The elementary modification is defined as a channel change on one of the flows or a power level change on one of the nodes. The pseudo code of our algorithm is given in Algorithm 1.

The algorithm starts with an initial "temperature" $T_{i n i}$ and an initial configuration $C_{i n i}$ with an initial power consumption $P_{i n i}$. It then looks for a neighbor 
configuration as the next configuration state, $C_{t e m p}$. After a neighbor is found, the algorithm first checks if the delay delay $y_{i}$ of every data flow under the neighbor configuration meets the delay constraint $D$. If the constraint is met, the algorithm calculates the power consumption difference, $\Delta P$, at the neighbor state and the current state. However, if the constraint is violated, the algorithm adds a Penalty to $\Delta P$. The Penalty is a parameter that needs to be tuned for the experiment in order to get a good solution. It helps the algorithm to avoid being trapped at a local minimum. The algorithm then checks if the power consumption is reduced. If the power consumption is reduced, the neighbor configuration is accepted. However, if the neighbor configuration causes an increased $\Delta P$ for power consumption, the algorithm calculates a probability by the exponential expression $e^{-\frac{\Delta P}{T}}$ and accepts the neighbor configuration based on this probability. After each iteration, the "temperature" is decreased by a factor of $\rho$. The algorithm ends when the "temperature" is smaller than the threshold $T_{\text {end }}$.

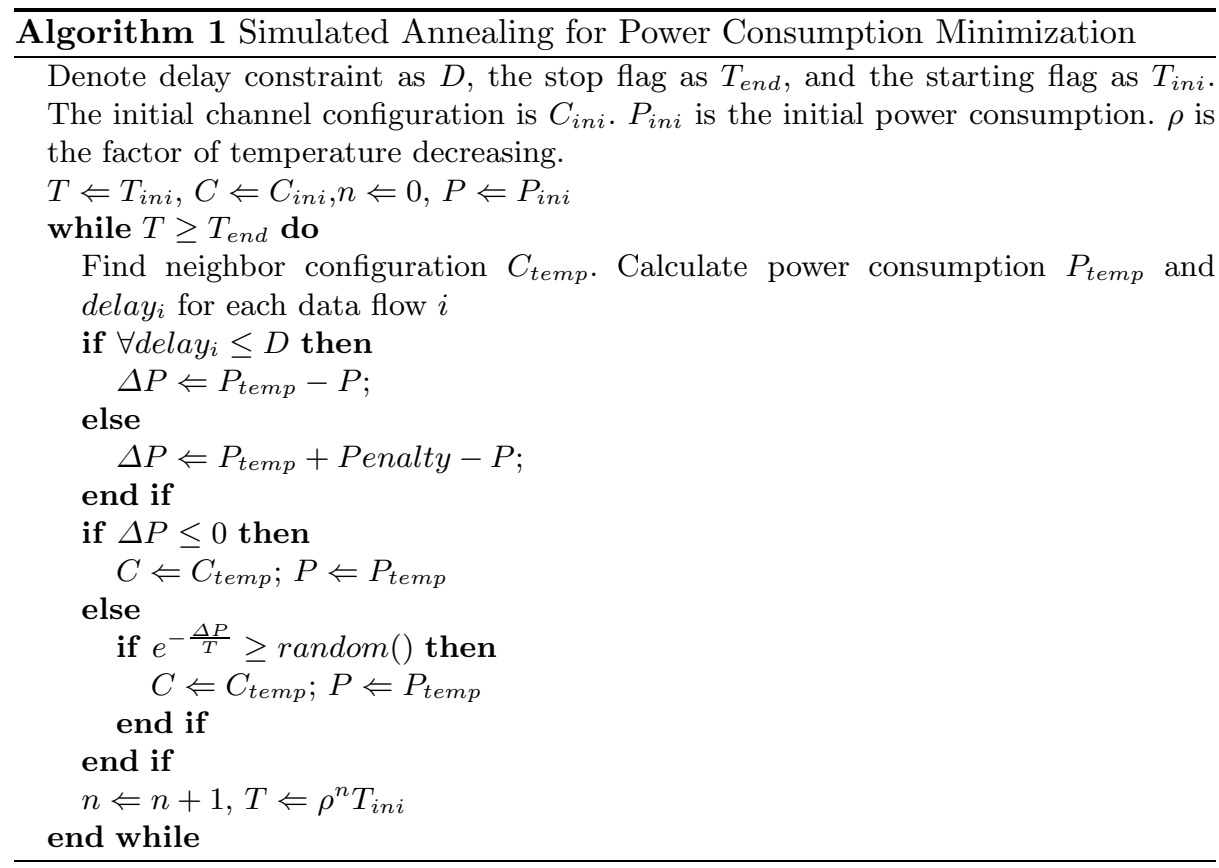

\section{Empirical Results}

In this section, we present the evaluation results of our configuration algorithm on a hardware testbed.

\subsection{Testbed Setup and Baselines}

Our testbed consists of 25 Tmote motes. Two different topologies used for the experiments are shown in Figure 5. Node 13, as the base station, consists of 5 real motes in the experiment, which emulates a super node with 5 radios. 
Independent uniform random traffic generator are implemented on each source node.

In the RSS measurement phase, every mote in the network takes turn to act as the sender and broadcasts packets using different power levels on different channels. While the sender is sending packets at a certain power level on a fixed channel $c$, all other nodes, acting as listeners, iterate through channel $c-1, c$ and $c+1$, and record the received signal strength on each channel. The reason we choose three channels to listen is because only

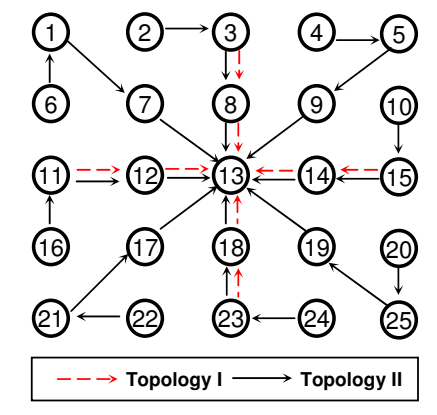

Fig. 5. Topologies used in experiments. the same channel and adjacent channels show the approximate linear PRR-Power pattern, as discussed in Section 3. In the experiments, we choose 5 discrete power levels: 3, 10, 17, 24 and 31, as the transmission power for the model establishment. This helps us to reduce the solution search space and speed up the experiments. After collecting all the RSS measurements, we import the data to the Simulated Annealing optimization program we implemented in MATLAB to compute the channel and power configuration.

We choose the following two baselines for comparison. The first baseline, called Orthogonal, uses only orthogonal channels for channel assignment and computes the power configuration by Simulated Annealing. The second baseline, called Random, also uses Simulated Annealing to find the desired power level for each node, but randomly assigns overlapping channels to flows. We use two metrics to evaluate the performance of these three protocols. The first metric is average end-to-end transmission count, which evaluates the end-to-end delay performance. The second metric is transmission power consumption per packet, which is the ratio between total transmission power and the number of packets transmitted. This metric evaluates the energy efficiency performance.

\subsection{Different Delay Constraints}

We first evaluate the three schemes under different transmission count constraints. In this experiment, we use Topology I in Figure 5 with nine motes, forming 4 flows. Three channels, 16, 17 and 18, are used. Channels 16 and 18 are orthogonal channels while channel 17 overlaps with channels 16 and 18 . Figure 6 shows the average end-to-end delay under different constraints. The overlapping scheme achieves a smaller average end-to-end transmission count than the two baseline schemes. In addition, the delay of our overlapping scheme is closest to the constraints. The reason for the superior performance of our overlapping scheme is that it takes advantage of overlapping channels with carefully selected power and channel configuration by the Simulated Annealing algorithm to reach suboptimal solutions. With more channel resources to use, the overlapping scheme achieves a better configuration solution than the other two 

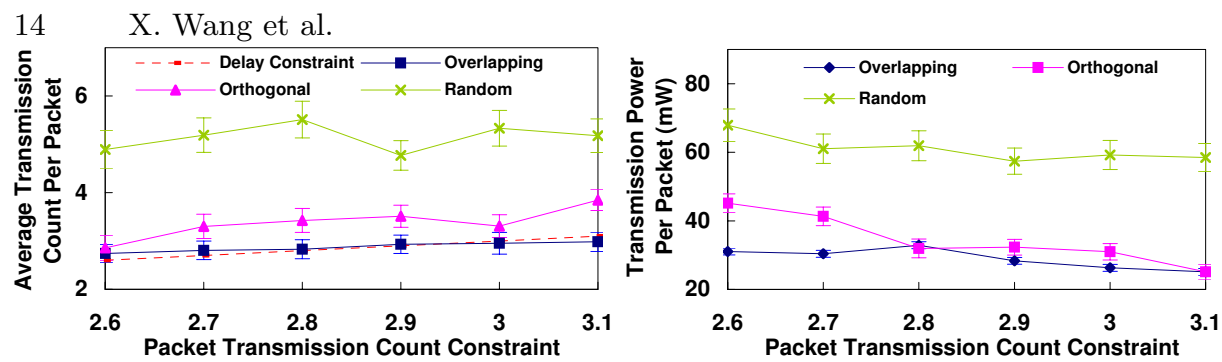

Fig. 6. Delay under different end-to-end Fig. 7. Power consumption under different transmission delay constraints end-to-end transmission delay constraints.

protocols. When the constraint becomes looser, all the schemes yield higher endto-end transmission counts. The results demonstrate that the end-to-end delay in the network is adaptive to the change of the delay constraint.

Figure 7 shows the transmission power consumption per packet for different constraints. Among all three schemes, the overlapping scheme consumes the least transmission power. This is because the overlapping scheme utilizes all the available channel resources and carefully chooses the most appropriate transmission power to reduce the interference among nodes such that the power consumed by retransmissions is significantly reduced. When the constraint is greater than 2.8, the performance of Orthogonal is close to that of our scheme. However, when the constraint is tight, Orthogonal performs significantly worse than the overlapping scheme. All the three schemes show decreasing trends for power consumption when the end-to-end transmission constraint becomes looser. This is because when the constraint is looser, we have a larger search space for the SA algorithm, likely resulting in a better power configuration.

\subsection{Different Flow Numbers}

Figures 8 and 9 show the performance of the network with different numbers of data flows. It is important to evaluate the performance of the network under different numbers of flows because multiple flows may need to share channels when the number of flows increases. In these experiments, we use Topology II in Figure 5, where 25 motes are organized as a 5 by 5 grid. The base station is placed in the center, similar to the previous two experiments. Each data flow has three hops and we gradually increase the number of data flows in the network from 5 to 8 . We use 5 overlapping channels, from channel 16 to channel 20, where 3 channels are orthogonal.

Figure 8 shows that the average transmission count per packet increases when the number of data flows increases. This is because more data flows cause more interferences in the network and more flows need to share the same channels for data transmissions, which results in more intra-channel interferences and competition. The same trend can be observed in Figure 9 for power consumption. Among all the three schemes, the overlapping scheme performs best for both the average transmission count and average power consumption. This is because the overlapping scheme utilizes most channel resources to reduce the reuse of each channel, which leads to less intra-channel interference. In the meantime, the 

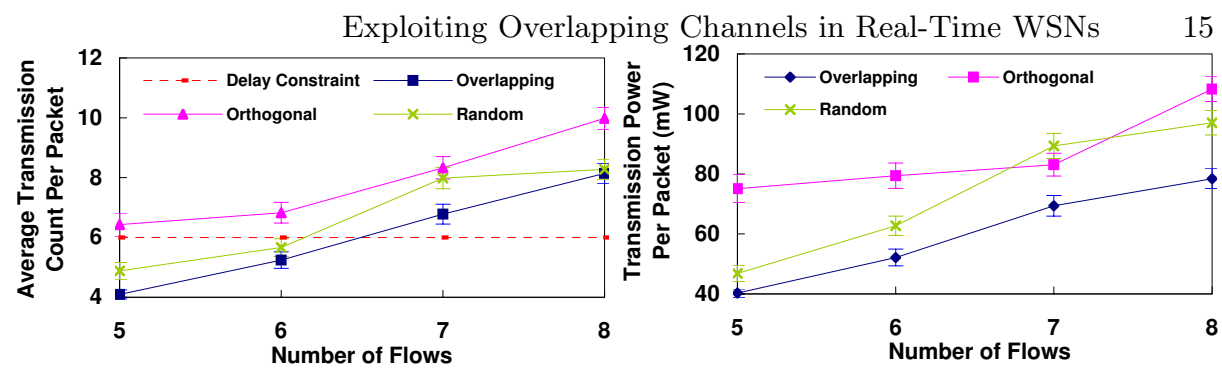

Fig. 8. Delay under different numbers of Fig. 9. Power consumption under different data flows. numbers of data flows.

overlapping scheme also carefully configures the transmission power to reduce the interferences among adjacent channels. Note that Orthogonal performs the worst because a greater number of flows need to share channels when there are only 3 orthogonal channels available. The increased channel sharing leads to a higher degree of channel competition and intra-channel interferences, and thus more packet retransmissions.

\section{Conclusions}

In this paper, we have conducted empirical studies to investigate the interferences among overlapping channels. Our results show that overlapping channels can also be utilized for improved real-time performance if the transmission power is carefully configured. In order to minimize the overall power consumption of a network with multiple data flows under end-to-end delay constraints, we formulate a constrained optimization problem to configure the transmission power level for every node and assign overlapping channels to different data flows. Since the optimization problem has an exponential computational complexity, we then present a heuristic algorithm designed based on Simulated Annealing to find a suboptimal solution. Our extensive empirical results on a 25-mote testbed demonstrate that our algorithm reduces both the end-to-end communication delay and overall transmission power consumption, compared with two baselines: a scheme using only orthogonal channels and a scheme using simple policy to assign overlapping channels.

\section{References}

1. "Cc2420 $2.4 \mathrm{ghz}$ ieee 802.15.4 / zigbee-ready rf transceiver." [Online]. Available: http://www.chipcon.com

2. P. Gupta and P. R. Kumar, "The capacity of wireless networks," IEEE Transactions on on Information Theory, vol. 46, no. 2, 2000.

3. J. Zhang, G. Zhou, C. Huang, S. H. Son, and J. A. Stankovic, "TMMAC: An energy efficient multi-channel mac protocol for ad hoc networks," in ICC, 2007.

4. G. Zhou, C. Huang, and et al., "MMSN: Multi-frequency media access control for wireless sensor networks," in INFOCOM, April 2006.

5. X. Wang, X. Wang, X. Fu, G. Xing, and N. Jha, "Flow-based real-time communication in multi-channel wireless sensor networks," in EWSN, 2009. 
6. Y. Wu, J. Stankovic, T. He, and S. Lin, "Realistic and efficient multi-channel communications in dense sensor networks," in INFOCOM, 2008.

7. H. Liu, H. Yu, X. Liu, C.-N. Chuah, and P. Mohapatra, "Scheduling multiple partially overlapped channels in wireless mesh networks," in ICC, 2007.

8. Z. Feng and Y. Yang, "Scheduling multiple partially overlapped channels in wireless mesh networks," in WCNC, 2008.

9. A. H. M. Rad and V. W. Wong, "Partially overlapped channel assignment for multi-channel wireless mesh networks," in ICC, 2007.

10. M. Sha, G. Xing, G. Zhou, S. Liu, and X. Wang, "C-mac: Model-driven concurrent medium access control for wireless sensor networks," in INFOCOM, 2008.

11. M. Demirbas and Y. Song, "An rssi-based scheme for sybil attack detection in wireless sensor networks," in WoWMoM, 2006.

12. J. A. Stankovic, T. Abdelzaher, C. Lu, L. Sha, and J. Hou, "Real-time communication and coordination in embedded sensor networks," Proceedings of the IEEE, vol. 91, no. 7, 2003.

13. M. Caccamo, L. Y. Zhang, and L. Sha, "An implicit prioritized access protocol for wireless sensor networks," in RTSS, 2002.

14. T. He, J. Stankovic, C. Lu, and T. Abdelzaher, "SPEED: A stateless protocol for real-time communication in sensor networks," in ICDCS, 2003.

15. G. Xing, M. Sha, J. Huang, G. Zhou, X. Wang, and S. Liu, "Multi-channel interference measurement and modeling in low-power wireless networks," in RTSS, 2009.

16. I. Talzi, A. Hasler, S. Gruber, and C. Tschudin, "Permasense: investigating permafrost with a wsn in the swiss alps," in EmNets, 2007.

17. A. Goldsmith, Wireless Communications. Cambridge University Press, 2005.

18. K. Karenos and V. Kalogeraki, "Real-time traffic management in sensor networks," in RTSS, 2006.

19. A. Mainwaring, D. Culler, J. Polastre, R. Szewczyk, and J. Anderson, "Wireless sensor networks for habitat monitoring," in ACM Workshop on Sensor Networks and Applications, 2002.

20. M. Maimour, "Maximally radio-disjoint multipath routing for wireless multimedia sensor networks," in WMuNep, 2008.

21. J. Deng, R. Han, and S. Mishra, "Decorrelating wireless sensor network traffic to inhibit traffic analysis attacks," in In Elsevier Pervasive and Mobile Computing Journal, Special Issue on Security in Wireless Mobile Computing Systems, 2006.

22. O. Chipara, Z. He, G. Xing, Q. Chen, X. Wang, C. Lu, J. Stankovic, and T. Abdelzaher, "Real-time power-aware routing in sensor networks," in IWQoS, 2006.

23. T. He, P. Vicaire, T. Yan, Q. Cao, G. Zhou, L. Gu, L. Luo, R. Stoleru, J. A. Stankovic, and T. F. Abdelzaher, "Achieving long-term surveillance in vigilnet," in INFOCOM, April 2006.

24. S. Kirkpatrick, C. D. G. Jr., and M. P. Vecchi, "Optimization by simulated annealing," Science, vol. 220, no. 4598, pp. 671-680, 1983.

25. T. M. Nabhan and A. Y. Zomaya, "A parallel simulated annealing algorithm with low communication overhead," IEEE Trans. Parallel Distrib. Syst., 1995.

26. L. Selavo, A. D. Wood, Q. Cao, T. Sookoor, H. Liu, A. Srinivasan, Y. Wu, W. Kang, J. A. Stankovic, D. Young, and J. Porter, "Luster: wireless sensor network for environmental research," in SenSys, 2007.

27. J. Jeong, D. E. Culler, and J.-H. Oh, "Empirical analysis of transmission power control algorithms for wireless sensor networks," EECS Department, University of California, Berkeley, Tech. Rep. UCB/EECS-2005-16, Nov 2005. 